\title{
COMUNICACIÓN
}

\section{TÉCNICAS QUIRÚRGICAS PARA LA REPARACIÓN DE LAS ÚLCERAS PROFUNDAS DE CÓRNEA}

\author{
Cristina Meneses B. ${ }^{1}$ y Mario Serna A. ${ }^{2}$
}

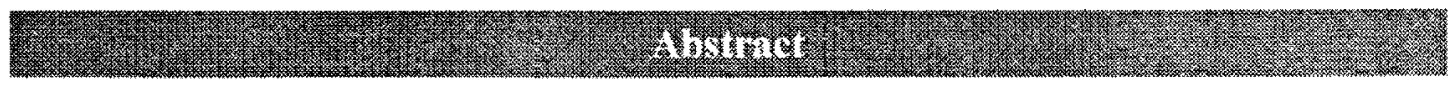

The cornea is formed of 4 layers: epithelium, estroma, descemet membrane and endothelium. Corneal ulcers are characterized by loss of the epithelium and a variable amount of estroma. Ulcers usually respond well to medical treatment and tend to heal, but rapidly progressing ulcers do not respond and require surgery. Some ulcers become deep, reaching the descemet membrane and producing descemetocele, a critical condition that is difficult to cure. Urgent surgical treatrent is required due to the risk of perforation because the Descemet membrane and the corneal endothelium are the only barrier to the aqueous humor. We treated 16 canines and 2 felines presenting profound corneal ulcers and descemetocele. They were divided into 4 groups, each treated differently, in order to determine the most best surgical technique. Variations in anesthesia time, kind of surgery and postoperative evolution are described.

Key words: Corneal ulcer, descemet membrane.

Palabras clave: Úlcera corneal, membrana de descemet.

Las úlceras de córnea se pueden clasificar según su profundidad en: Superficiales, afectando solamente el epitelio (tratamiento médico) y profundas, incluyendo el epitelio, estroma y la membrana de descemet sin llegar a perforarla (tratamiento quirúrgico) (Barnett, 1992; Gelatt, 1999; Morgan y Abrams, 1994; Nasisse, 1997 y Stades, 1999).

El diagnóstico de úlcera se realiza con el uso de fluoresceína, colorante hidrosoluble que no tiñe la córnea normal porque no atraviesa el epitelio hidrofóbico. Si el epitelio está incompleto la fluoresceína penetra en el estroma corneal hidrofílico y lo colorea de

\footnotetext{
'Encargada del Área Especializada de Oflalmología - Facultad de Veterinaria - Montevideo - Uruguay

"Director Técnico de Veterinaria "Grito de Gloria"y "Su-can». Montevideo-Uruguay. Email: crismar@ adinet.com.uy
}

verde claro (Brooks, 1999; Gelatt, 1999; Slatter, 1992 y Stades, 1999).

Cuando en las úlceras corneales profundas el fondo no se colorea, está indicando que la membrana de descemet está incluida en la cavidad ulcerada, que por su carácter hidrofóbico le impide la toma del colorante. La membrana de descemet es muy frágil, tiene de 3 a 12 micromilímetros de espesor, es elástica y con buen potencial para las infecciones, si se perfora hay salida del humor acuoso, prolapso de iris y pérdida de la visión. (Gelatt, 1999; Millichamp, $1990 \mathrm{y}$ Slatter, 1992).

Las técnicas seleccionadas para el tratamiento de estas úlceras son: flap del tercer párpado, flap de $180^{\circ}$, flap de $360^{\circ}$ y flap pediculado (Eisner, 1990; Kuhn, 1979; Peiffer et al., 1977 y Scagliotti, 1988). Mediante el tratamiento quirúrgico se aporta tejido de sos- 
tén (conjuntivo) a la úlcera para que a partir de éste se regenere el epitelio y repare (Naumann y Sautter, 1988 y Scagliotti, 1988).

Se trataron 16 caninos y dos gatos a los cuales se les diagnosticó úlceras de córnea profundas con descemetocele.

Al examen oftalmológico completo se descartaron animales que presentaban otras patologías oculares asociadas como aumento de la presión intraocular, queratoconjuntivitis seca, queratitis pigmentaria $y$ panoftalmitis, para no interferir con el proceso de cicatrización.

Las intervenciones quirúrgicas se realizaron con anestesia general inhalatoria, instrumental de cirugía general y oftalmológico. El protocolo anestésico fue el siguiente: Preanestesia con Acetilpromazina $0.01 \mathrm{mg} / \mathrm{k}, \mathrm{l} /$ $\mathrm{V}$, Sulfato de atropina $0.5 \mathrm{mg} . / \mathrm{k}, 1 / \mathrm{V}$ y antibióticos de amplio espectro, $\mathrm{I} / \mathrm{M}, 30 \mathrm{mi}-$ nutos antes de la cirugía. Diazepan $10 \mathrm{mg}, \mathrm{V} /$ $\mathrm{V}$ como miorrelajante, Ketamina $10 \mathrm{mg} . / \mathrm{k}, \mathrm{I} /$ $V$ para la inducción y anestesia inhalatoria con Isoflorano para el mantenimiento. Los pacientes se colocaron en decúbito lateral con el ojo a intervenir hacia arriba, realizándose el lavado de los sacos conjuntivales utilizando iodóforos y suero fisiológico. Se colocaron los campos operatorios y blefaróstatos realizando la limpieza de la úlcera y retirando todo el material que se encontraba suelto, tejido necrosado, exudados, etc., pasando un cottonete con solución iodófora por el borde la úlcera para reavivar el tejido (Barnett, 1992; Brooks, 1999; Gelatt, 1999 y Slatter, 1992).

Los grupos formados según las distintas técnicas fueron:

Grupo 1: Se realizó flap de tercer párpado y sutura palpebral. Se coloca un gel antibiótico sobre la úlcera y se extiende el tercer párpado fijándolo mediante dos o tres puntos de sutura sobre la conjuntiva bulbar en la parte supero-lateral del globo ocular. Luego se cierran los párpados mediante puntos simples separados (blefarorrafia). Si el ojo es muy prominente como en el caso de animales braquicéfalos: pequinés, boxer, shih-tzu, estos puntos pueden hacerse capitoneados para darles más resistencia evitando los desgarros de la conjuntiva. Total de animales intervenidos: 10, tiempo de la cirugía: 20 minutos en promedio.

Grupo 2: Cirugía en flap de $180^{\circ}$ mediante la disección de parte de la conjuntiva bulbar desde el borde corneal que se desplaza cubriendo la úlcera, luego se suturan los párpados. Total de animales cuatro, tiempo promedio de cirugía: 30 minutos.

Grupo 3: Flap de $360^{\circ}$ disecando toda la conjuntiva bulbar en la perisferia de la córnea, se desplaza cubriendo la córnea y suturando en la línea media y los párpados. Animales intervenidos: 2 , tiempo promedio de cirugía: de 30 a 40.

Grupo 4: Flap pediculado con un trozo de conjuntiva bulbar de $2 \mathrm{~cm}$. aproximadamente, que se diseca en la región dorsolateral del globo ocular unido por su base a la conjuntiva, en su extremo libre se sutura a los bordes de la úlcera en forma circular mediante puntos separados, hilo 8-0 bajo magnificación y se suturan los párpados. A los 10 días se abren los párpados y bajo anestesia tópica y sedación se corta la unión del pedículo con la conjuntiva. Se continúa con tratamiento médico hasta la total integración de la conjuntiva con la córnea. Total de animales 1 , tiempo promedio de cirugía: 60 minutos.

Durante el postoperatorio todos los animales fueron tratados con antibióticos en forma de pomada dos veces por dia. Se realizaron lavados con suero y siempre se utilizó collar isabelino. Los puntos de los párpados se retiraron luego de 10 días, observándose en todos los casos un edema de córnea cuya evolución depende del tipo de cirugía que se haya realizado.

Grupos 1: Al retirar los puntos del tercer párpado no se observaron adherencias con 
el epitelio de la córnea y las úlceras cicatrizaron correctamente con tejido de neoformación vascularizado. Los edemas desaparecieron después de la primera semana de retirados los puntos.

Grupos 2 y 3: Las cirugías en los cuales se utilizó la conjuntiva bulbar para el tratamiento del descemetocele dejaron importantes uniones entre la conjuntiva bulbar y el epitelio de la córnea (adherencias) que se vieron en el momento de retirar los puntos. La cicatrización de la úlcera se realizó correctamente pero las adherencias y el edema demoraron más tiempo en resolverse.

Grupo 4: Esta técnica requiere la intervención de un cirujano especialista y los tiempos anestésicos son prolongados. Se necesita equipamiento adecuado y magnificación, el edema no es tan importante como en los casos anteriores, pero se requirió más control en el tratamiento postoperatorio y vigilancia del pedículo hasta obtener una correcta unión con el tejido a rellenar (de 15 a 21 días).

Es importante hacer un diagnóstico correcto con la utilización de fluoresceína para evaluar la profundidad de la úlcera. Cuando las úlceras son superficiales (fluoresceína positiva) el tratamiento no tiene porque ser quirúrgico, pudiendo ser solamente médico. En aquellos casos en que frente a una úlcera nos da un resultado fluoresceína negativa (descemetocele), el único tratamiento indicado es el quirúrgico y además es una urgencia oftalmológica donde hay riesgo de ruptura ocular y pérdida de la visión. Estamos frente a un caso grave por lo que debemos actuar con rapidez

La técnica de corrección de las úlceras por medio del flap de tercer párpado es una técnica sencilla y fácil de realizar que nos brinda excelentes resultados, se lleva a cabo en tiempos anestésicos cortos y no requiere equipamiento especializado. $\mathrm{El}$ instrumental es el utilizado en cirugía general y requiere menor destreza que los otros métodos.

En este caso las úlceras cicatrizan más rápido debido a que las lágrimas de la glándula del tercer párpado aportan la humedad y los nutrientes necesarios para facilitar la cicatrización (Gelatt, 1999; Petersen y Crispin, 1999 y Slatter, 1992). 


\section{FOTOS Y FIGURAS SOBRE ÚLCERAS DE CÓRNEAS Y TÉCNICAS QUIRÚRGICAS PARA LA REPARACIÓN}

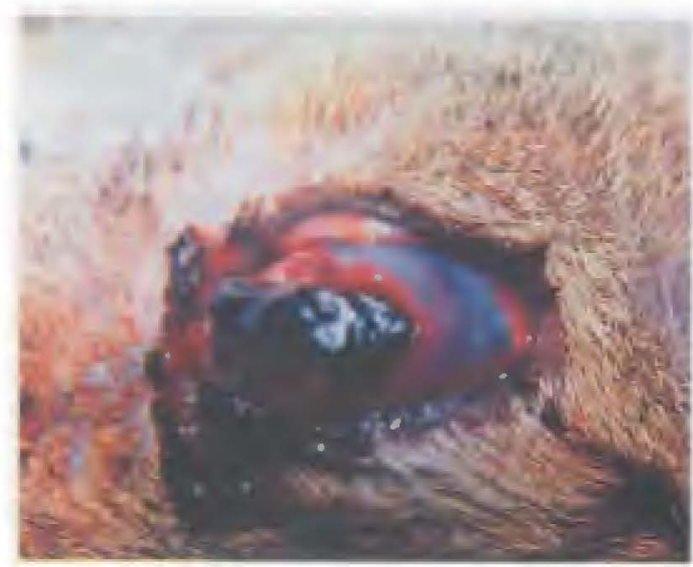

Felino de 3 años con ulcera en ojo izquierdo perforada

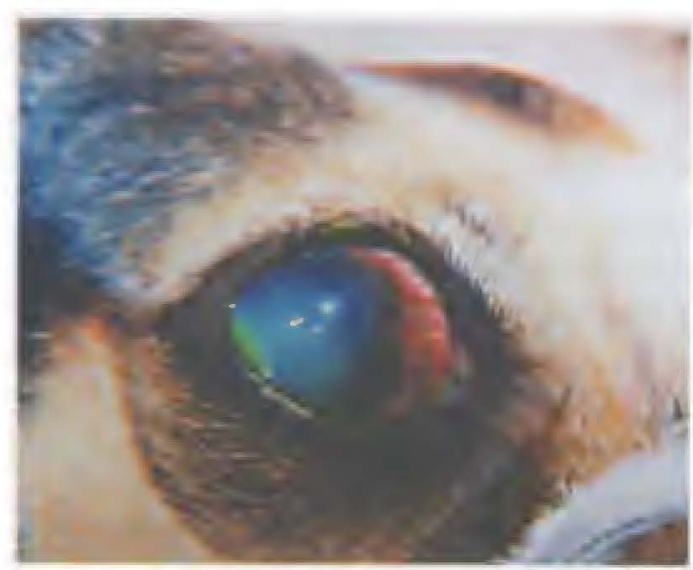

Canino macho de 8 años con ulcera coloreada con fluoresceína positiva.

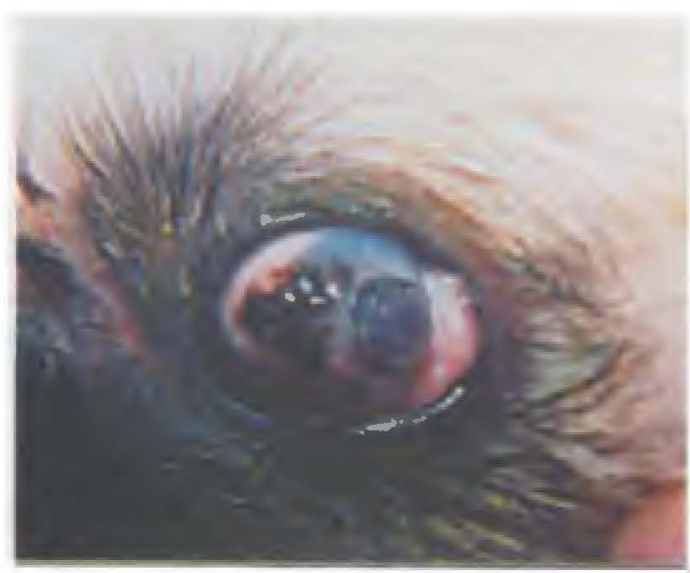

Pequines de 6 años con desceme-tocele y queratitis pigmentaria.

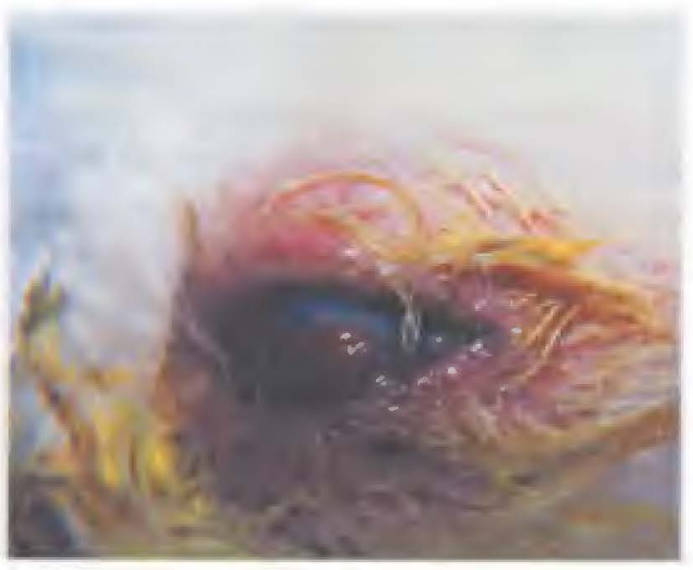

White Poodle dos años con descemetocle

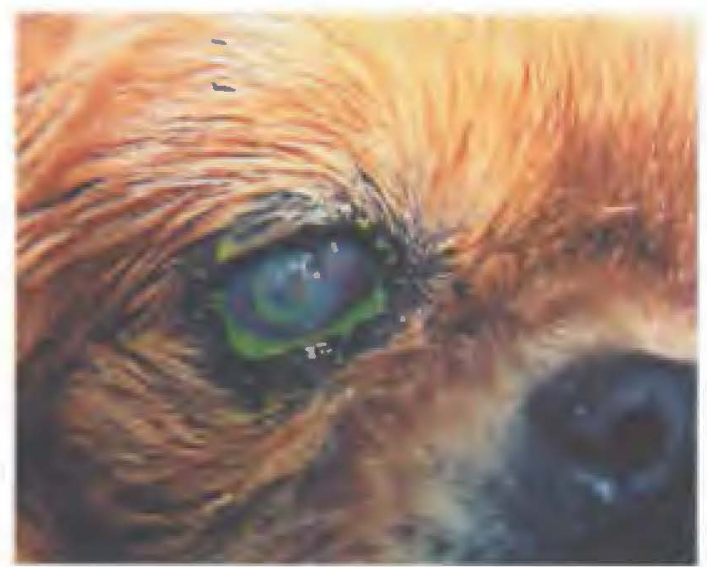

Canino pequines de 6 años ulcera profunda, descemetocele fluoresceína negativo.

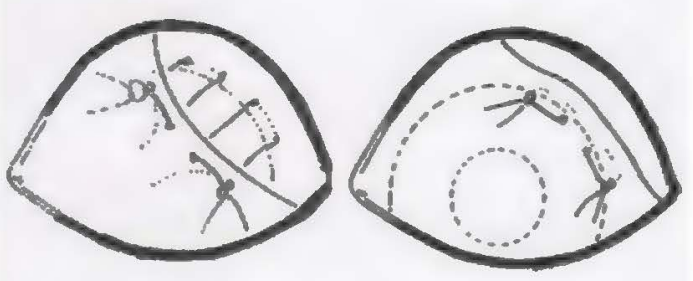

Flap de tercer párpado. 

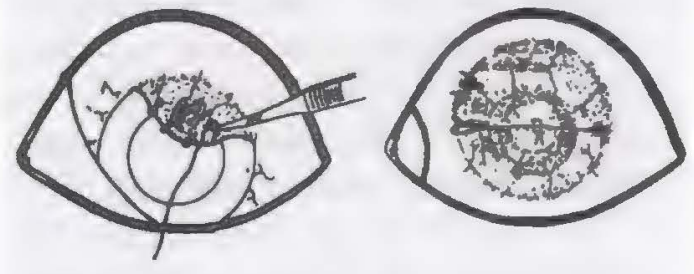

Flap de $180^{\circ}$ y Flap de $360^{\circ}$
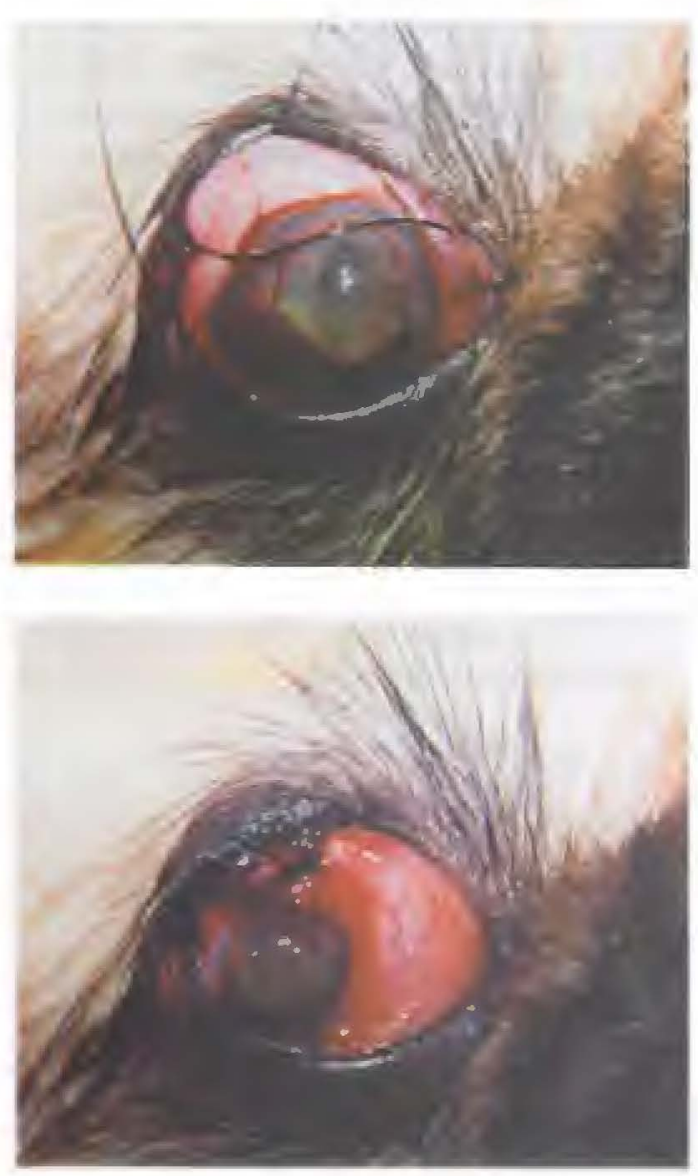
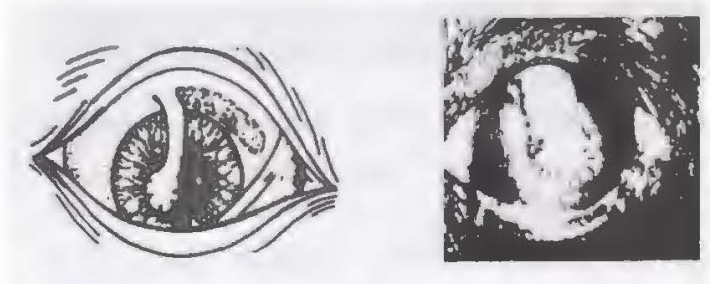

F.syuema y foto de Flap pediculado

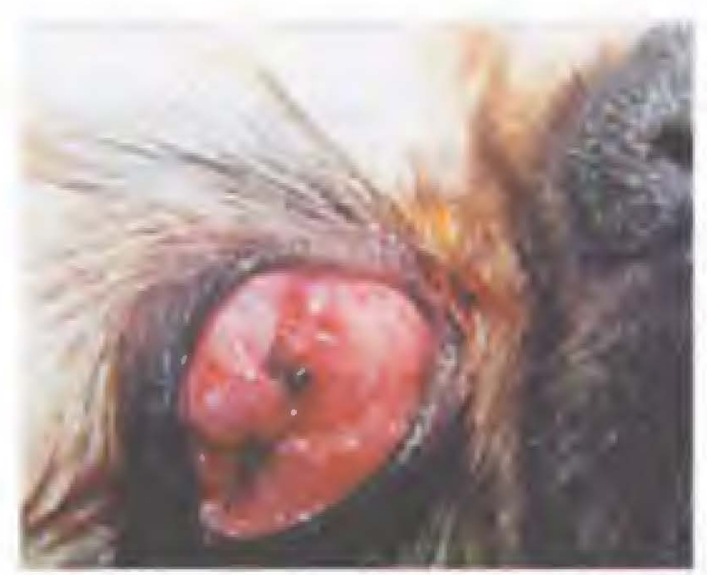

Pequines de 6 años con descemetocele técnica del tercer párpado

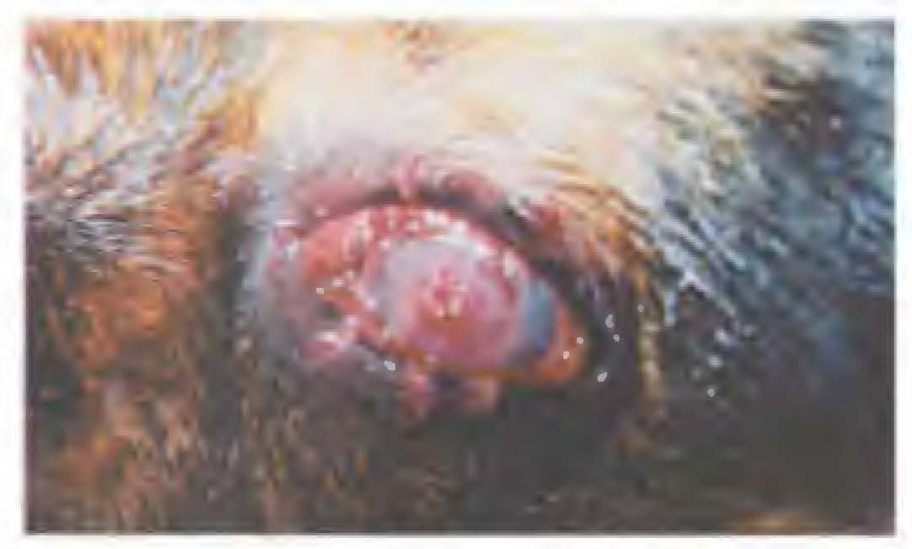

Canino cruza con adherencias luego de retirados los puntos 


\section{Ufertura Otwh}

1. Barnett, K.C. 1992. Veterinary ophthalmology atlas. Pág. 39. Grass ediciones - London.

2. Brooks, D. 1999. Current concepts in veterinary ophthalmology. 7:21-24. USA.

3. Eisner, G 1990. Suturing the cornea and sclera. In: Eisner. G. Ed. Eye surgery: an introduction to operative technique $2^{\text {nd }}$ Ed. Berlin: Springer - Verlag 182190. Alemania.

4. Gelatt, K. 1999. Veterinary Ophthalmology 3d. edition. Lippincott Williams \$ Wilkins. 20:680-688. USA.

5. Kuhn, E.L. 1979. Conjunctival patch grafts for treatment of corneal lesions in dogs-Mod. Vet. Pract. 31:301-305. USA.

6. Millichamp, N. 1990. The veterinary Clinics of North America - Small animal practice 627-642. USA.

7. Morgan, R. y K. Abrams. 1994. A comparison of six different therapies for persistent comeal erosions in dogs and cats -4:38-43. Vet. Comp. Ophthalmol. USA.
8. Nasisse, M. 1997. Clínicas Veterinarias de Norte América - Clínica de pequeños animales. Pág. 1110-1113. USA.

9. Naumann, G y H. Sautter. 1988. Surgical procedures on the cornea. Pág. 433508. In: Blodi., F, Mackensen, G. Neubauer, H. Eds. Surgical Ophthalmology - Berlin: Springer-Verlag.

10. Peiffer, R.; K. Gelatt y R.Gwin. 1977. Tarsoconjuntival pedicle grafts for deep ulceration in the dog and cat $-\mathrm{J}$. Am. Anim. Hosp. Assoc. 13:387-391. USA

11. Petersen, S. y S. Crispin. 1999. Manual de oftalmología en pequeños animales. 7:154-164. Harcourt Brace. London.

12. Scagliotti, R. 1988. Tarsoconjuntival island grafts for the treatment of deep corneal ulcers, desmetocoeles and perforations in 35 dogs and 6 cats - 3:69-76. Semin. Vet. Med. Surg. Small Anim. USA.

13. Slatter, D. 1992. Fundamento de oftalmología veterinaria. 11:345-353. Intermédica. USA.

14. Stades, F. 1999. Oftamología para el veterinario práctico. Pág. 117-118. Intermédica. Alemania. 